\title{
Amélioration de la durée de conservation des bananes plantain aux températures ambiantes
}

\author{
Alexis Nicaise LEPENGUE ${ }^{1^{*}}$, Isaac MOUARAGADJA ${ }^{1}$, Emmanuel DICK ${ }^{2}$, \\ Bertrand MBATCHI ${ }^{1}$ et Séverin AKE ${ }^{2}$ \\ ${ }^{1}$ Université des Sciences et Techniques de Masuku, (USTM) Unité de Recherche Agrobiologie ; BP : 067 \\ Franceville, Gabon. \\ ${ }^{2}$ Université de Cocody - Abidjan, Laboratoire de Physiologie Végétale, Agrophysiologie ; 22 \\ $B P$ : 582 Abidjan 22. \\ *Auteur correspondant, E-mail : lepengue_nicaise@yahoo.fr, Tel. : (00242) 067647 38/0768 4362.
}

\section{RESUME}

La banane plantain est le premier aliment de base au Gabon. Malheureusement 30 à $40 \%$ de sa production sont perdues, par manque de techniques de conservation appropriées et accessibles à la majorité des producteurs. Le présent travail a été conçu pour expérimenter l'efficacité de quelques procédés traditionnels de conservation, susceptibles de servir d'alternative aux atmosphères modifiées. Il s'agit notamment de l'emballage des fruits en sachets de polyéthylène, de leur enrobage dans de l'huile d'arachide, et de leur enfouissement dans la vermiculite. Les résultats ont révélé que les bananes ensachées se conservaient significativement plus longtemps que celles traitées à l'huile d'arachide, ou enfouies dans la vermiculite. De faibles pertes de masse et d'intégrité tissulaire, accompagnées de légères augmentations de sucres réducteurs ont également été observées chez ces organes. Les combinaisons sachet-huile et sachet-vermiculite n'ont pas provoqué des rallongements significatifs des durées de conservation, comparativement au sachet utilisé seul. Les emballages plastiques permettent donc d'améliorer la conservation des fruits de bananes plantain.

(C) 2010 International Formulae Group. All rights reserved.

Mots clés : Banane plantain, conservation, sachet, intégrité, Gabon

\section{INTRODUCTION}

Le bananier plantain est l'une des principales plantes alimentaires de base des pays en voie de développement dont le Gabon est le $19^{\mathrm{e}}$ exploiteur africain, avec une production de 0.27 tonnes/année (FAO, 2004). Trente à $40 \%$ de cette production sont malheureusement perdus avant la consommation, en raison principalement des mauvaises conditions de récolte, de transport et de conservation (Lépengué, 1999). Concernant la conservation, de nombreux travaux ont permis de proposer des solutions appropriées, notamment l'entreposage d'organes à des températures comprises entre $8{ }^{\circ} \mathrm{C}$ et $12{ }^{\circ} \mathrm{C}$, et la modification de l'atmosphère intérieure des entrepôts (Varoquaux et al., 2002). Les travaux de Narayana et al. (2002) ont par exemple montré que les bananes plantain (cultivar Karpuravalli) enveloppées dans des emballages plastiques hermétiques et stockées aux températures inférieures à $13,5{ }^{\circ} \mathrm{C}$ allongeaient leur durée de vie verte de 19,33 
jours. En Chine, Chen et al. (2000) ont montré que le prétraitement des bananes plantain par lavage à l'eau chlorée et leur emballage dans des sacs en polyéthylène augmentaient leur durée de conservation plus de 60 jours en chambre froide (aux températures de 10-15 $\left.{ }^{\circ} \mathrm{C}\right)$. D'autres techniques de conservation sont couramment utilisées en Occident : réduction des teneurs d' $\mathrm{O}_{2}$ et augmentation de celles du $\mathrm{CO}_{2}$ dans les entrepôts; réfrigération des organes par glaçage, brumage ou air forcé; modulation des pressions de vapeur, ou des vitesses de refroidissement des enceintes frigorifiques etc. (Chamara et al., 2000; Varoquaux et al., 2002). Ces méthodes quoiqu'efficaces restent onéreuses et hors de portée de nombreux exploitants qui constituent la majeure partie des producteurs gabonais. Le problème reste donc presqu'entier, et la nécessité de concevoir de nouvelles techniques pratiques et accessibles à l'ensemble des paysans s'avère donc incontournable. Dans le présent travail nous testons l'efficacité de quelques procédés traditionnels de conservation, avec l'espoir de suppléer les stockages en enceintes réfrigérées. Les différentes techniques expérimentées sont à base de sachets plastiques, d'huile d'arachide et de vermiculite.

\section{MATERIEL ET METHODES Matériel}

Le matériel d'étude est constitué de fruits de bananier plantain cultivar Corne 1 (triploïde $\mathrm{AAB}$, issu du croisement Musa parasitica x Musa acuminata), récoltés sur des bananiers des champs paysans périphériques à la ville de Franceville.

\section{Traitement des bananes}

Des régimes de bananes récoltés 80 jours après l'émergence de l'axe inflorescentiel ont été précautionneusement transportés au laboratoire, préservés de toutes blessures. Les fruits d'environ $300 \mathrm{~g}$ ont été sélectionnés et désinfectés par trempage pendant $5 \mathrm{~min}$. dans une cuve contenant 50 $\mathrm{dm}^{3}$ d'hypochlorite de sodium (1\%), et desséchés entre 2 épaisseurs de papier Joseph (Lépengué, 1999). Les bananes ont subi 6 traitements différents : 1) emballage dans des sachets en polyéthylène de marque Bibby, étanches et transparents d'épaisseurs $0,10 \mathrm{~cm}$; 2) immersion pendant 30 secondes dans $1 \mathrm{dm}^{3}$ d'huile d'arachide; 3) enfouissement des bananes dans de la vermiculite contenue dans un carton de $45 \mathrm{dm}^{3}$ de volume. Les trois autres traitements ont consisté à combiner ces paramètres deux à deux. Pour chaque traitement, 6 doigts ont été utilisés, ce qui correspond à 36 organes, pour les 6 traitements effectués. Six (6) bananes non traitées ont été conservées dans les conditions ambiantes du laboratoire $\left(25^{\circ} \mathrm{C}\right)$, pour servir de témoins. Tous les 10 jours, un organe a été retiré et soumis à l'analyse de chacun des paramètres morphométriques et biochimiques décrits ci-dessous.

\section{Evaluation des paramètres morphologiques et biochimiques \\ Durée de conservation}

Pour chaque traitement, la durée de conservation des bananes a été évaluée par le temps s'écoulant entre le début d'incubation et l'apparition des premiers symptômes de nécrose tissulaires manifestée par les taches de noircissement des peaux des organes.

\section{Perte de masse}

La perte de masse (\% M) des bananes a été évaluée après pesée tous les 10 jours à l'aide d'une balance de marque Sartorius, par la formule suivante :

$$
\% \mathrm{M}=\frac{\mathrm{Mi}-\mathrm{Mt}}{\mathrm{Mi}} \times 100 \text {, }
$$

Mi : Masse initial de la banane

Mt : Masse de la banane à un temps défini

\section{Perte de résistance mécanique}

La perte de résistance mécanique des organes a été mesurée par application d'un pénétromètre numérique (de marque Kochler, $10 \mathrm{~kg}$ ), sur la partie médiane du fruit. La perte de résistance à un instant déterminé a été calculée à partir de la résistance initiale, suivant le modèle de l'équation de perte de masse [1]. 


\section{Perte d'intégrité}

La perte d'intégrité des bananes a été mesurée à partir de la conductivité des tissus de leurs épidermes. Dix (10) rondelles de 5 $\mathrm{mm}$ de diamètre de chaque organe ont pour cela été prélevées à l'aide d'un emporte-pièce, immergées dans $10 \mathrm{ml}$ d'eau distillée désionisée, et incubées en agitation continue (agitateur Vibramax ; $50 \mathrm{rpm}$ ) pendant 1 heure (Lépengué et al., 2008). Les solutions ont ensuite été filtrées sur papier Watham $\mathrm{n}^{\circ} 2$, et leur conductivité mesurée à l'aide d'un conductimètre de type Jenway Electrochemestry. Les pertes de conductivité ont été calculées à partir des valeurs initiales, selon le modèle d'équation présenté en [1].

\section{Teneur en sucres réducteurs}

Les sucres réducteurs ont été extraits après broyage de $2 \mathrm{~g}$ de pulpe de banane dans $10 \mathrm{ml}$ d'éthanol $80^{\circ} \mathrm{GL}$, et centrifugation pendant 10 minutes à $15000 \mathrm{rpm}$. Les culots ont été repris dans $10 \mathrm{ml}$ d'éthanol $40^{\circ} \mathrm{GL}$, et centrifugés à nouveau comme précédemment. Les surnageants ont été additionnés et purifiés successivement avec $5 \mathrm{ml}$ d'oxaloacétate (10\%) et $5 \mathrm{ml}$ d'acétate de plomb (10\%). L'alcool a ensuite été éliminé par évaporation sur un bain de sable chauffant à $80{ }^{\circ} \mathrm{C}$ (Lépengué et al., 2008). Les sucres réducteurs de l'extrait glucidique ont été dosés par colorimétrie au spectrophotomètre (Jenway 6310 Spectrophotometer) à $540 \mathrm{~nm}$ avec l'acide dinitrosalicylique de sodium (Lépengué et al., 2009).

\section{Répétitions et traitement statistique}

Chaque expérience mentionnée dans ce travail a été répétée 3 fois, et les résultats soumis à une analyse de variance au logiciel Statistica 6.0. Les tests comparatifs de Newman-Keuls ont été utilisés, en cas de différence significative, au seuil de 5\%.

\section{RESULTATS}

Effet des traitements sur la durée de conservation des bananes

Les résultats de l'effet de la vermiculite, de l'huile d'arachide, du sachet et de leurs différentes combinaisons sur la durée de conservation des bananes plantain ont été résumés à la Figure 1. Leur analyse a révélé que tous les 6 traitements provoquaient un allongement significatif de la durée de conservation des organes. Les meilleurs résultats ont été obtenus avec le sachet utilisé seul $\left(55 \%\right.$ au $60^{\mathrm{e}}$ jour), en association avec l'huile d'arachide (58\% au $60^{\mathrm{e}}$ jour) ou avec la vermiculite $\left(57 \%\right.$ au $60^{\mathrm{e}}$ jour). En revanche les allongements de plus faibles durées ont été notés chez les bananes conservées dans la vermiculite $\left(15 \%\right.$ au $60^{\mathrm{e}}$ jour) ou enrobées d'huile d'arachide (19\% au 60 jour).

L'analyse statistique a montré que les effets induits par l'huile et la vermiculite n'étaient pas significativement différents. Par ailleurs, l'addition d'un traitement supplémentaire (huile ou vermiculite) aux sachets n'augmentait pas significativement la durée de conservation des bananes.

\section{Effet de différents traitements sur la perte de masse des organes}

Les études de l'effet de vermiculite de l'huile, du sachet et de leurs diverses combinaisons sur la perte de masse des bananes plantain ont donné des résultats présentés à la Figure 2. De leur analyse, il est ressorti que tous ces traitements atténuaient significativement la perte de masse des bananes. Les plus faibles valeurs ont été observées chez les organes ensachés simplement, ou en association avec la vermiculite ou l'huile. Au $60^{\mathrm{e}}$ jour, pour ces 3 traitements, les baisses de masse étaient toutes inférieures à 10\%, alors que celles des témoins dépassaient largement $45 \%$. Les traitements les moins efficaces ont été notés avec la vermiculite (30\%) et l'huile d'arachide $(25 \%)$, au $60^{\mathrm{e}}$ jour de conservation.

\section{Effet des différents traitements sur la résistance mécanique}

L'étude de la résistance mécanique des bananes plantain ayant subi les traitements à la vermiculite, à l'huile d'arachide et au sachet a généré des résultats présentés à la Figure 3. D’une façon générale, les différents traitements ont significativement réduit les 
pertes de fermeté des organes étudiés, tendant ainsi à maintenir leur consistance intacte. Les réductions les plus importantes ont été obtenues chez les bananes ensachées (simplement), ou en combinaison avec de l'huile et de la vermiculite. Au bout de 60 jours, les pertes dues à ces 3 traitements sont restées inférieures à $20 \%$, alors que celles des témoins approchaient les taux de $97 \%$.

\section{Effet de différents traitements sur la perte d'intégrité}

Les résultats de cette étude ont révélé que les différents traitements expérimentés ralentissaient significativement les pertes d'intégrité des bananes, au cours de leur stockage (Figure 4). Les plus fortes réductions ont été observées chez les bananes préalablement enrobées d'huile d'arachide et ensachées, avec des valeurs de perte d'intégrité inférieures à $20 \%$, au $60^{\mathrm{e}}$ jour. En revanche, les plus faibles réductions ont été notées chez les bananes respectivement traitées à l'huile d'arachide (63\%), et à la vermiculite $(76 \%)$, pour la même durée de conservation.

\section{Effet sur la teneur des sucres réducteurs}

Les résultats de ce travail ont montré que les bananes ayant subi les différents traitements à l'huile, à la vermiculite, au sachet et à leurs diverses combinaisons présentaient des teneurs en sucres réducteurs significativement inférieures à celles des organes témoins (Figure 5). La formation de ces composés se faisaient lentement chez les organes traités jusqu'au $40^{\mathrm{e}}$ jour d'incubation, avant d'augmenter rapidement pour atteindre au $60^{\mathrm{e}}$ jour des valeurs comprises entre 18 $\mathrm{mg} / \mathrm{g}$ de matière fraîche, pour le traitement huile-sachet, et $74 \mathrm{mg} / \mathrm{g}$ de matière fraîche pour celui de la vermiculite. Chez les organes témoins, ces valeurs seuils $(74 \mathrm{mg} / \mathrm{g}$ ) étaient pratiquement déjà atteintes dès le $20^{\mathrm{e}}$ jour de conservation.

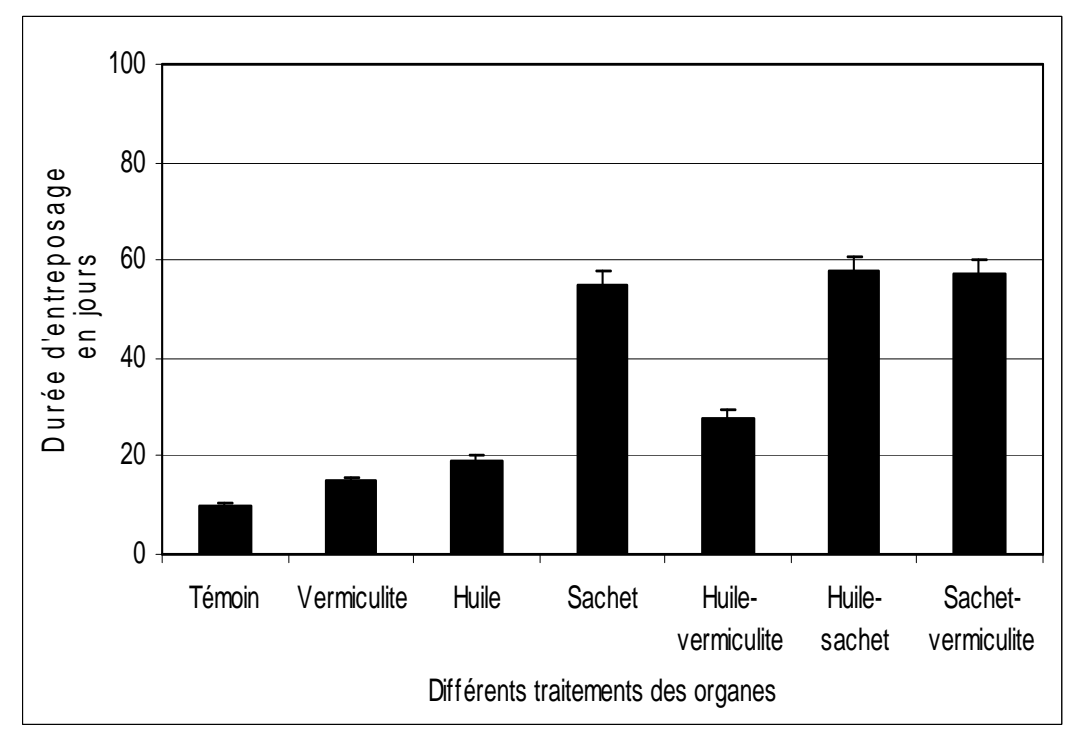

Figure 1 : Durée de conservation des fruits de bananes plantain soumis à différents traitements, à la température de $25^{\circ} \mathrm{C}$. 


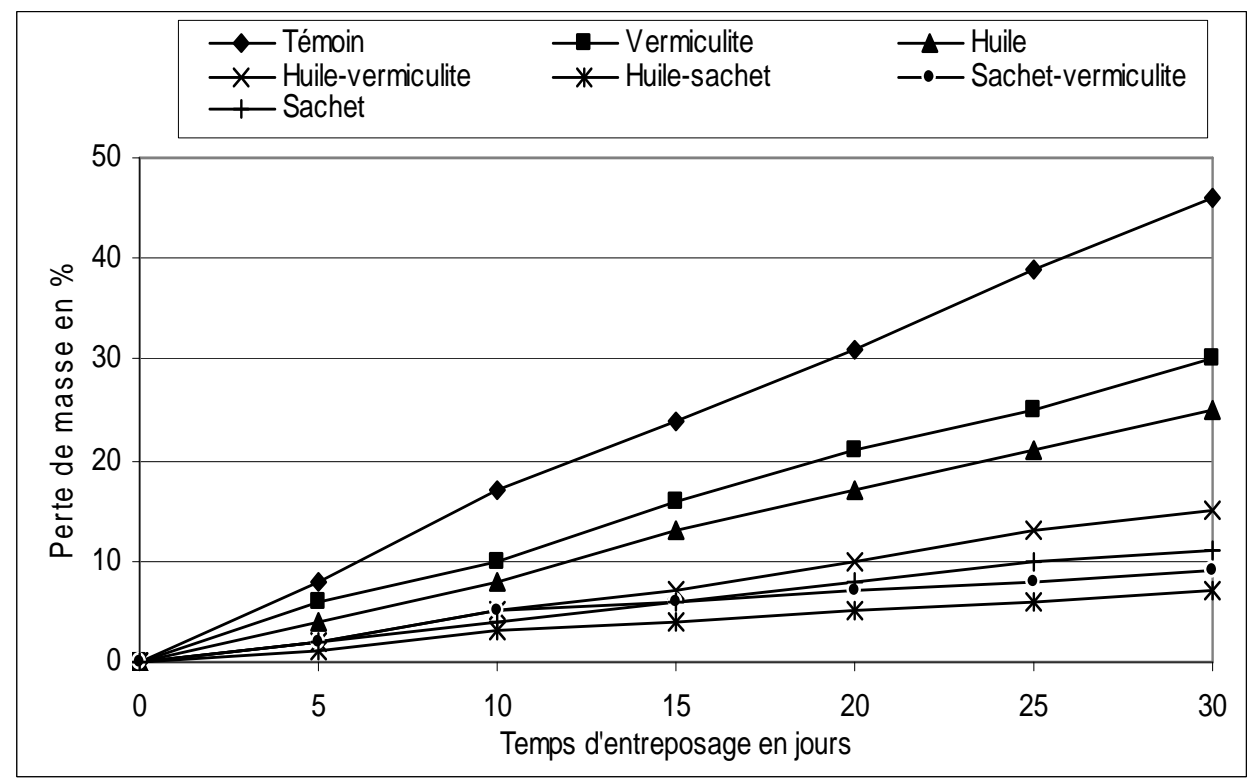

Figure 2 : Perte de masse des fruits de bananes plantain soumis à différents traitements de conservation, à la température de $25^{\circ} \mathrm{C}$.

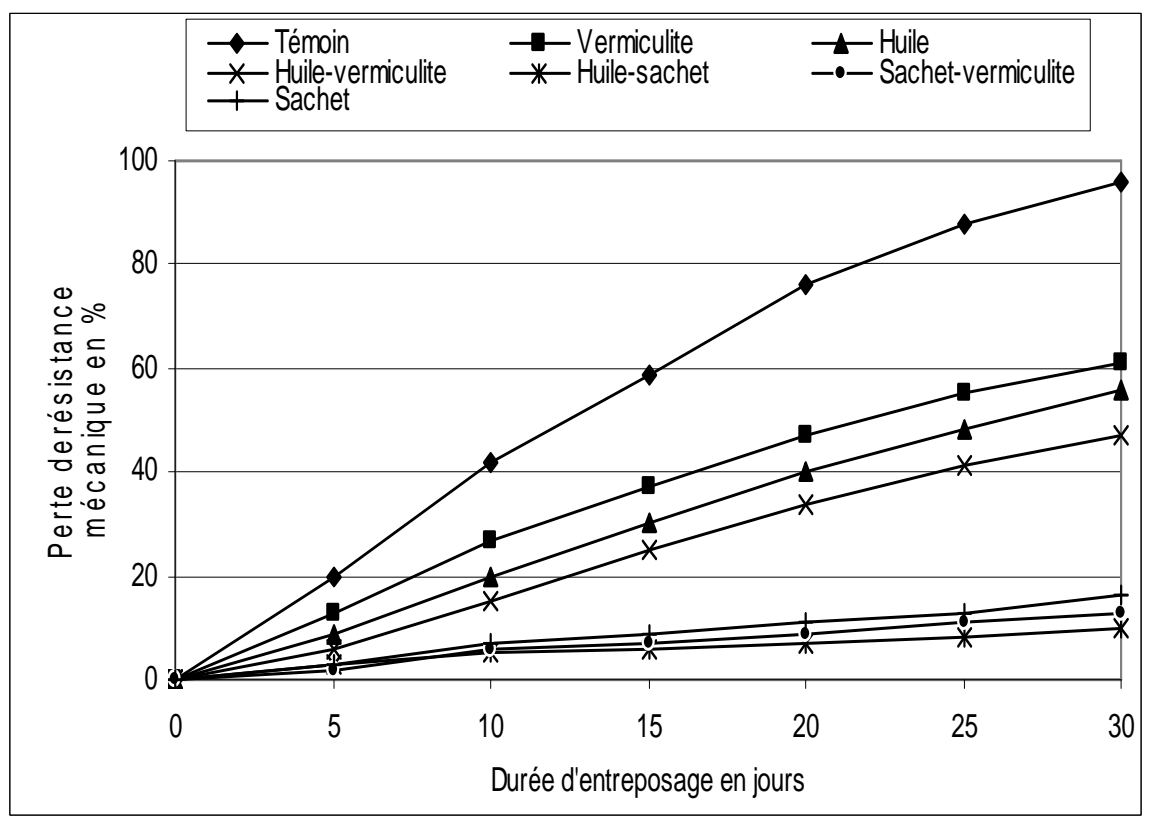

Figure 3 : Perte la consistance des fruits de bananes plantain soumis à différents traitements de conservation, à la température de $25^{\circ} \mathrm{C}$. 


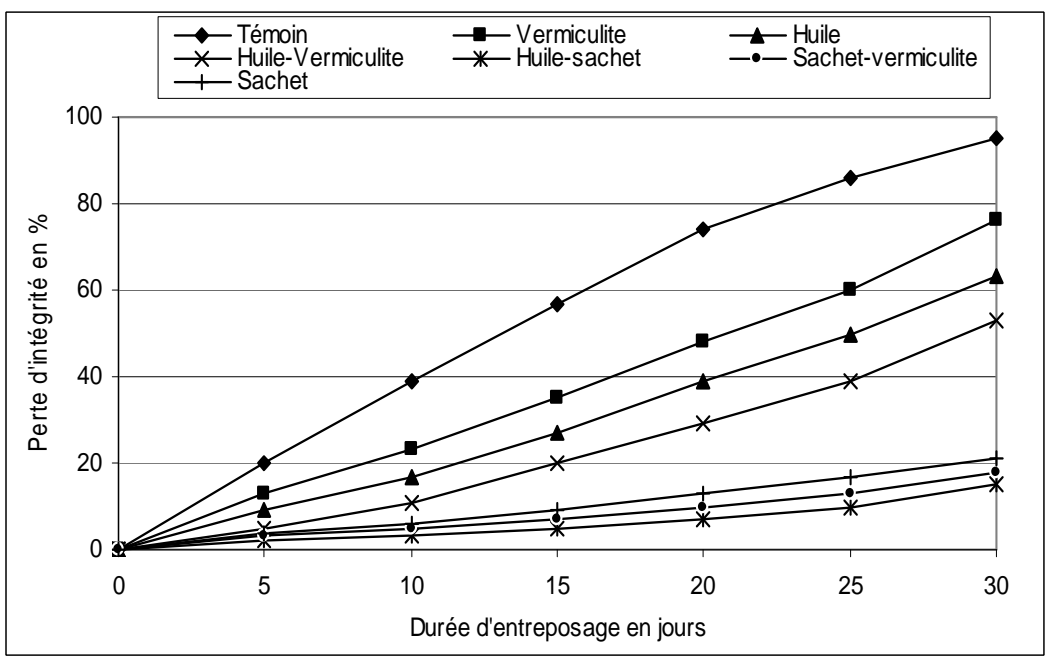

Figure 4 : Perte d'intégrité des fruits de bananes plantain soumis à différents traitements de conservation, à la température de $25^{\circ} \mathrm{C}$.

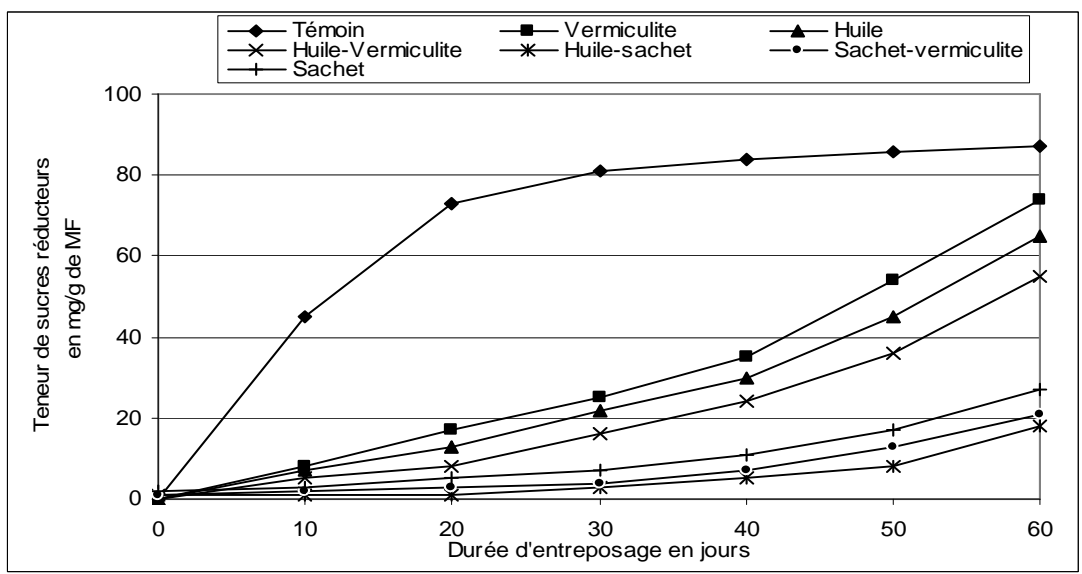

Figure 5 : Evolution des sucres réducteurs chez les fruits de bananes plantain soumis à différents traitements de conservation, à la température de $25^{\circ} \mathrm{C}$.

\section{DISCUSSION}

Les résultats de ce travail ont, dans l'ensemble, montré que les différents traitements (vermiculite, huile, sachet et leurs différentes combinaisons) influençaient significativement les paramètres morphologiques et physiologiques des bananes en conservation. On a notamment relevé un prolongement de la vie verte des organes, la conservation de leur fermeté et intégrité, et la faiblesse des taux de leurs sucres réducteurs. Toutes ces méthodes utilisées en milieu traditionnel constituent donc (même si elles ne peuvent substituer les atmosphères contrôlées) un moyen véritablement avantageux de conservation des organes, en comparaison aux conditions ambiantes. Des résultats similaires ont été rapportés par Dick (2006) à propos de l'effet des emballages en sachets et des membranes 
en silicone sur la conservation des bananes en Côte d'Ivoire. Cet auteur a en effet montré que la durée d'entreposage de ces organes était significativement allongée (plus de 20 jours), après leur traitement au sachet ou au silicone. Les travaux de Karunaratne et al. (2002) traitant les bananes plantain avec les solutions d'acide acétique $(0,2 \%)$ et de vinaigre $(0,2 \%)$ ont abouti aux mêmes conclusions.

A l'analyse de tous ces résultats, les modifications morpho-physiologiques et biochimiques observées paraissent découler d'une même origine: la perturbation des échanges gazeux entre les organes et le milieu d'entreposage. En effet, toutes les techniques utilisées (enrobage d'huile, enfouissement, ensachage), sont basées sur le même principe, à savoir la conservation par isolément des organes de leur milieu ambiant. Ce qui correspond pratiquement à des conditions d'atmosphère contrôlée, au sens défini par Narayana et al. (2002). Ces dispositions conduisent à des modifications des équilibres gazeux, et plus précisément à l'épuisement de l'oxygène (par la respiration), et à l'accumulation du $\mathrm{CO}_{2}$, dans le milieu. Les basses tensions d'oxygène conduisent à des réductions de l'activité respiratoire, et au ralentissement de nombreuses activités métaboliques cellulaires (Varoquaux et al., 2002). Chez les dattes dorées (Phoenix dactylifera) conservées en conditions d'oxygène appauvri (1\%) par exemple, des baisses de réactions d'oxydation de la chlorophylle à l'origine du jaunissement doré de ces fruits ont été observées par Moshen et Naoufel (2005). Les travaux de Leitsner et Gould (2002) ont aussi montré que, chez les fraises et les pêches conservées avec 2\% d'oxygène, les activités de nombreuses enzymes de dégradation des acides organiques (malique, succinique, tartrique...) conduisant aux arômes fruités caractéristiques du mûrissement étaient fortement réduites, prolongeant ainsi l'état vert de ces organes.
L'action du $\mathrm{CO}_{2}$ est également jugée favorable à la conservation des fruits et légumes en atmosphère contrôlée (Dupin, 1992; Chamara et al., 2000). Ce gaz est, en effet connu pour son action inhibiteur sur les enzymes de la chaîne respiratoire, et surtout pour son opposition à l'action de l'éthylène, phytohormone stimulatrice du mûrissement des fruits. Les études de ces auteurs ont par ailleurs montré que le $\mathrm{CO}_{2}$ freinait également l'activité des enzymes de la chaîne de dégradation de l'amidon notamment, les phosphorylases, les phosphohexoisomérases, et les phosphofructokinases, rabaissant ainsi la quantité des sucres réducteurs produits, et donc la saveur sucrée des organes. Toutes ces actions physiologiques conduisent au ralentissement du métabolisme et donc au prolongement de la conservation des fruits (Varoquaux et al., 2002).

Le revêtement des organes d'une membrane ou d'une couche superficielle réduit aussi l'impact de la chaleur et du vent sur les organes entreposés, conduisant à la baisse de la transpiration et de l'évaporation, et donc à celle des pertes de poids (Varoquaux et al., 2002). Les épidermes des fruits conservent ainsi une bonne quantité d'humidité qui tend à garder les organes frais. C'est l'une des raisons qui expliquerait la faible perte de poids des bananes traitées, comparativement aux témoins. Des résultats similaires ont été rapportés par Narayana et al. (2002) sur les bananes stockées en emballages de sachets hermétiques.

\section{Conclusion}

La conservation des bananes en sachet, dans la vermiculite, ou enrobées d'huile permet de prolonger significativement leur vie verte. Ce qui donne des organes verts, fermes, avec de légères pertes de poids et de solutés, ainsi que de faibles productions de sucres réducteurs. Les meilleurs résultats sont toutefois obtenus avec des organes ensachés (en traitement seul, ou associé avec de l'huile 
ou de la vermiculite). Ce mode de conservation simple et pratique, quoique rudimentaire, constitue donc une alternative exploitable, en absence de conditions modernes d'enceintes réfrigérées.

\section{REFERENCES BIBLIOGRAPHIQUES}

Chamara D, Illeperuma K, Theja Galappatty P, Sarananda KH. 2000. Modified atmosphere packaging of 'Kolikuttu' bananas at low temperature. Journal of Horticultural Science and Biotechnology, 75(1): 92-96.

Chen W, Wu Z, Su M, Zhu J. 2000. Technologie post-récolte, transport et commercialisation des bananes en Chine. Agrociencia (Mexique), 36(2): 169-180.

Dick E. 2006. Contribution à l'étude de la physiologie du mûrissement, de l'entreposage et de la conservation de la banane plantain après récolte. Thèse d'Etat, Université de Cocody-Abidjan, $243 \mathrm{p}$.

Dupin H. 1992. Alimentation et Nutrition Humaines. Edition ESF : Paris, France; $1533 \mathrm{p}$.

FAO. 2004. Données de la FAOSTAT. Rapport $\quad \mathrm{N}^{\circ} \quad 1$ sur l'Afrique. www.fao.org/docrep/006/J1954F/J1954f0 4.htm

Karunaratne AM, Ethugala CV. 2002. État sanitaire, changements physicochimiques et goût des bananes traitées avec de l'acide acétique ou du vinaigre. Fruits, 57(1): 11-18.

Leitsner P, Gould J. 2002. Technologies combinées de conservation des fruits et des légumes. Archives de Documents de la FAO. www.fao.org/docrep/006/ J1954F/J1954f04.htm
Lépengué AN. 1999. Activités enzymatiques et troubles de maturation des bananes plantain entreposées aux températures élevées. Diplôme d'Etudes Approfondies, en Physiologie Végétale (Agrophysiologie), UFR Biosciences, Université de Cocody-Abidjan, 68 p.

Lépengué AN, M'batchi B, Aké S. 2008. Production, caractérisation, et utilisation des composés toxiques de Phoma sabdariffae Sacc. dans la sélection des cultivars résistants de roselle (Hibiscus sabdariffa L. var. sabdariffa) au Gabon. Agronomie Africaine, 20(1): 59-67.

Lépengué AN, Mouaragadja I, M'batchi B, Aké S. 2009. Etude de quelques caractéristiques physico chimiques du filtrat toxique de Phoma sabdariffae Sacc., agent pathogène de la roselle. Sciences et Nature, 6(2): 95-105.

Moshen A, Naoufel B. 2005. Effet des conditions d'entreposage sur la dégradation de la couleur des dattes tunisiennes de type Deglet-Nour. Fruits, 60(1): 41-46.

Narayana CK, Mustafa MM, Sathiamoorthy S. 2002. Influence de l'emballage et du stockage sur la durée de vie et la qualité des bananes de la variété Karpuravalli. Indian Journal of Horticulture, 59(2): 113-117.

Varoquaux P, Gouble B, Ducamp MN, Self G. 2002. Méthode permettant d'optimiser l'emballage des fruits sous atmosphère modifiée. Fruits, 57(5-6): 313-322. 